\title{
Are radiographs needed when MR imaging is performed for non-acute knee symptoms in patients younger than 45 years of age?
}

\author{
Bert P. M. ter Braak • Patrice W. J. Vincken • \\ Arian R. van Erkel • Rolf M. Bloem • L. J. Napoleon • \\ M. N. Coene • Peter A. van Luijt • Sam de Lange • \\ Johan L. Bloem
}

Received: 29 May 2007 / Revised: 31 July 2007 / Accepted: 25 August 2007 / Published online: 3 October 2007

(C) ISS 2007

\begin{abstract}
Objective The objective was to determine the value of radiographs in young adults with non-acute knee symptoms who are scheduled for magnetic resonance imaging (MRI). Materials and methods Nine hundred and sixty-one consecutive patients aged between 16 and 45 years with knee symptoms of at least 4 weeks' duration were prospectively included in three participating hospitals. After applying exclusion criteria, 798 patients remained. Exclusion criteria were previous knee surgery (including arthroscopy) or
\end{abstract}

B. P. M. ter Braak · P. W. J. Vincken $(\varangle) \cdot$ A. R. van Erkel •

J. L. Bloem

Department of Radiology, Leiden University Medical Center, P.O. Box 9600, 2300 RC Leiden, The Netherlands

e-mail: p.w.j.vincken@lumc.nl

\section{R. M. Bloem}

Department of Orthopaedic Surgery,

Leiden University Medical Center,

Leiden, The Netherlands

L. J. Napoleon · M. N. Coene

Department of Orthopaedic Surgery, HAGA Hospital,

The Hague, The Netherlands

P. A. van Luijt

Department of Traumatology, Leiden University Medical Center,

Leiden, The Netherlands

S. de Lange

Department of Orthopaedic Surgery, Medical Center Haaglanden, The Hague, The Netherlands

Present address:

R. M. Bloem

Department of Orthopaedic Surgery, Reinier de Graaf Gasthuis,

Delft, The Netherlands
MRI, history of rheumatoid arthritis, clinical diagnosis of retropatellar chondromalacia, contra-indication for MRI and recent trauma. We identified two groups: group A with no history of trauma $(n=332)$, and group B with an old ( $>4$ weeks) history of trauma $(n=466)$. Patients had a standardized history taken, and underwent a physical exam, antero-posterior (AP) and lateral radiographs and MRI. We evaluated the radiographs and MRI for osseous lesions, articular surface lesions, fractures, osteoarthritis, loose bodies, bone marrow edema and incidental findings. Subsequently, patients with osseous abnormalities (Kellgren grade 1 and 2 excluded) on radiographs and a matched control group was evaluated again using MRI without radiographs.

Results Median duration of symptoms was 20 weeks. In group A, radiographs showed 36 osseous abnormalities in 332 patients (10.8\%). Only 13 of these, all Kellgren grade 1 osteoarthritis, were not confirmed on MRI. MRI showed 72 (21.7\%) additional abnormalities not confirmed on radiographs. In group B, radiographs showed 40 osseous abnormalities (8.6\%) in 466 patients. Only 15 of these, all Kellgren grade 1 osteoarthritis, were not confirmed on MRI. MRI showed 194 (41.6\%) additional abnormalities not confirmed on radiographs. The second evaluation of MRI without radiographs in 34 patients was identical to the first MRI evaluation. Common lesions were significantly more often diagnosed with MRI than with radiographs.

Conclusion Radiographs should not be obtained routinely when MRI is being performed in young adults with nonacute knee complaints because the yield and added value to MRI are low.

Keywords Knee $\cdot$ Magnetic resonance imaging . Radiographs 


\section{Introduction}

Radiographs in patients with acute knee problems that are obtained at, or close to, the time of trauma serve mainly to exclude fractures and most of these radiographs are normal. To reduce the total number, and especially the number of normal radiographs, clinical decision rules that identify patients with a high risk of fracture have been developed and validated [2-4]. In patients with non-acute knee symptoms radiographs are the mandatory minimal initial exam in patients with non-traumatic knee pain, according to ACR appropriateness criteria [5]. Magnetic resonance imaging (MRI) is frequently obtained if additional imaging is clinically needed to search for suspected intra-articular abnormalities. The value of radiographs relative to MRI in a heterogeneous group of patients was recently reported [6]. Radiographs were rated "essential" or "very important" in $46-58 \%$ of cases and added "not needed information" in $14-29 \%$ of cases. However, the value of radiographs relative to MRI in young adults presenting with non-acute knee symptoms has, to our knowledge, not been established. If in these patients radiographs could be omitted, or obtained only when needed when reading MRI, this could possibly lead to a significant reduction of the volume of knee radiographs with ensuing reduction in radiation dose and costs.

The purpose of our prospective study was to determine the value of radiographs in young patients with non-acute knee symptoms and suspected intra-articular pathology who are scheduled for MRI based on the results of a physical exam. We therefore determined the prevalence of osseous abnormalities visible on radiographs and MRI and the yield of MRI compared with that of radiographs. In addition, we measured the radiation dose of standard radiographs of the knee.

\section{Material and methods}

Over 3 years, 961 patients referred from general practitioners to the outpatient surgery or orthopedic surgery clinics of three hospitals (two general hospitals and one university hospital) because of non-acute knee symptoms agreed to participate in our prospective study. The symptoms had to have been present for at least 4 weeks. The institutional review board of each hospital approved the study. We obtained written informed consent from all patients. At study entry a standardized history, including inventory of traumatic events and other knee-related events such as morbidity and medical procedures was taken. Physical exam was performed by one of 15 (orthopedic) surgeons or by residents under their supervision. All the patients included underwent MRI and radiographs of the knee. Recent radiographs taken for the same complaints that were obtained prior to study inclusion were not repeated.

We included patients who met the following inclusion criteria: knee symptoms (pain, swelling of the joint, feeling of instability or giving way, history of locking) of at least 4 weeks' duration and age between 16 and 45 years. To obtain a homogenous study population without co-morbidity we did not include patients who had had previous surgery of the affected knee (including arthroscopy) or previous MRI, a history of rheumatoid arthritis, and clinical diagnosis of retropatellar chondromalacia, contra-indication for MRI, or an incomplete data set. One hundred and sixtythree patients $(17.0 \%)$ were eventually excluded (Table 1$)$, leaving 798 patients as our study group. Of these excluded patients a substantial number should not have been included by the orthopedic surgeons in the first place according to our study protocol, for instance, because they were either too young, too old or had knee complaints of less than 4 weeks' duration.

One out of six musculoskeletal radiologists (all with at least 5 years' experience) evaluated the radiographs without knowledge of the MR images and recorded their findings on a Case Record Form (CRF).

The radiation dose of standard antero-posterior and lateral radiographs was determined in two of the three hospitals using flat ionization chambers. We used PCXMC software (STUK, Finland) to convert the measured radiation doses into effective doses.

The radiologist evaluated the radiographs for articular surface lesions, using the classification developed by Bohndorf [7]. Osteoarthritis was scored using the scoring system developed by Kellgren et al. [1]. In the analysis, grades 1 to 3 were considered as one group, with osteoarthritis being present.

Radiographs were also evaluated for fractures [8-10], loose bodies, and incidental findings. A loose body was defined as a radiodense circumscriptive lesion located in the

Table 1 Excluded patients. Of the 961 patients referred, $163(17.0 \%)$ were excluded

\begin{tabular}{ll} 
Exclusion criteria & $n$ \\
\hline Symptoms of less than 4 weeks' duration & 65 \\
Aged under 16 years & 1 \\
Aged over 45 years & 6 \\
Previous surgery & 8 \\
Retropatellar chondromalacia & 14 \\
Previous MRI & 1 \\
Radiographs unavailable or not obtained & 65 \\
MRI or arthroscopy prohibited & 2 \\
Immediate arthroscopy required & 30 \\
\hline
\end{tabular}

Criteria are not mutually exclusive. 
Table 2 Osseous abnormalities detected on radiographs only, on radiographs and MRI, and on MRI only in 332 patients with non-acute nontraumatic knee complaints

\begin{tabular}{llllll}
\hline Osseous abnormality & $\mathrm{R}(\%)$ & $\mathrm{R}$ and MR $(\%)$ & MRI $(\%)$ & Total $(\%)$ & McNemar test, $p$ value \\
\hline OA & $13(3.9)$ & $18(5.4)$ & $39(11.7)$ & $70(21.1)$ & 0.00 \\
ASL & $0(0)$ & $3(0.9)$ & $7(2.1)$ & $10(3.0)$ & 0.02 \\
LB & $0(0)$ & $1(0.3)$ & $6(1.8)$ & $7(2.1)$ & 0.03 \\
Fractures & $0(0)$ & $0(0)$ & $0(0)$ & $0(0)$ & NA \\
Incidental finding & $0(0)$ & $1(0.3)$ & $0(0.0)$ & $1(0.3)$ & 1.00 \\
Bone marrow edema & $0(0)$ & $0(0)$ & $20(6.0)$ & $20(6.0)$ & NA \\
Total & $13(3.9)$ & $23(6.9)$ & $72(21.7)$ & $108(32.5)$ & 0.00 \\
\hline
\end{tabular}

OA: osteoarthritis grade 1 or higher according to Kellgren [1] on radiographs and according to Recht [14] on MRI ; ASL: articular surface lesion with or without disrupted cartilage; LB: loose body; R: abnormality detected with radiographs only; R and MR: abnormality detected with both radiograph and MRI; MRI: abnormality detected with MRI only; NA: not applicable. Categories are not mutually exclusive.

femorotibial, patellofemoral jointline or suprapatellar pouch. Incidental findings, such as non-ossifying fibromas and enchondromas were diagnosed using established criteria [11, 12]. On radiographs a lesion was considered a non-ossifying fibroma if it met the following criteria: asymptomatic, cortical metaphyseal location, and radiographically non-aggressive. A lesion was considered an enchondroma if it met the following criteria: central metaphyseal location, chondroid matrix, geographic pattern of destruction with or without sclerotic margin.

We performed the MR studies in all three hospitals on an identical 0.5-T system (Gyroscan T5, Philips Medical Systems, Best, the Netherlands), with the same software release using a dedicated transmit-receive knee coil.

The standardized scanning protocol consisted of three sequences: a sagittal and a coronal dual spin-echo (SE) sequence and a sagittal T1-weighted 3D gradient-echo (GE) sequence with frequency selective fat suppression. The following parameters were identical for both SE sequences: field of view $140-160 \mathrm{~mm}$, echo time (TE) $20 / 80 \mathrm{~ms}$. The coronal dual SE had a repetition time (TR) of $2,100 \mathrm{~ms}$, a $256 \times 205$ matrix and a slice thickness of $5 \mathrm{~mm}$ with a 0.5 $\mathrm{mm}$ interslice gap. The sagittal dual SE had a TR of $2,350 \mathrm{~ms}$, a $256 \times 179$ matrix and a slice thickness of $4 \mathrm{~mm}$ with a 0.4-mm interslice gap. The parameters for the sagittal frequency selective fat-suppressed T1-weighted
3D GE were: TR $70 \mathrm{~ms}$, TE $13 \mathrm{~ms}, 45^{\circ}$ flip angle, field of view $160 \mathrm{~mm}, 256 \times 205$ matrix, $4 \mathrm{~mm}$ slice thickness with a 2 -mm overlap.

The total imaging time of the standard protocol (including the initial survey sequence) was $26 \mathrm{~min}$.

After reading the radiographs one out of six radiologists evaluated MR images and reported his findings on a CRF. In accordance with usual management the observer had access to the radiographs, but not to the reports, when evaluating MR images. The CRF was similar to the one used for radiographs [8-12], except that bone marrow edema was added as an item [13]. For articular surface lesions we again used the classification developed by Bohndorf [7]. For osteoarthritis we used the scoring system developed by Recht et al. [14]. For the purpose of this study, grades 1 to 4 were considered as one group, with osteoarthritis being present.

To overcome bias in the reading of MR studies, introduced by knowledge of the results of conventional radiographs, one radiologist (JLB) re-evaluated MR studies of patients with abnormalities on conventional radiographs with the exception of osteoarthritis of Kellgren [1] grades 1-2. We used the same CRF format. At this second evaluation, MR studies of patients with osseous abnormalities on conventional radiographs were mixed with age- and gender-matched MR studies of patients without abnormal-

Table 3 Osseous abnormalities detected on radiographs only, on radiographs and MRI, and on MRI only in 466 patients with non-acute traumatic knee complaints

\begin{tabular}{|c|c|c|c|c|c|}
\hline Osseous abnormality & $\mathrm{R}(\%)$ & $\mathrm{R}$ and $\mathrm{MR}(\%)$ & MRI (\%) & Total $(\%)$ & McNemar test, $p$ value \\
\hline $\mathrm{OA}$ & $15(3.2)$ & $13(2.8)$ & $65(13.9)$ & $93(20.0)$ & 0.00 \\
\hline ASL & $0(0)$ & $3(0.6)$ & $22(4.7)$ & $25(5.4)$ & 0.00 \\
\hline LB & $0(0)$ & $1(0.2)$ & $4(0.9)$ & $5(1.1)$ & 0.13 \\
\hline Fractures & $0(0)$ & $4(0.9)$ & $3(0.6)$ & $7(1.5)$ & 0.25 \\
\hline Incidental finding & $0(0)$ & $4(0.9)$ & $2(0.4)$ & $6(1.3)$ & 0.5 \\
\hline Bone marrow edema & $0(0)$ & $0(0)$ & $96(20.6)$ & $96(20.6)$ & NA \\
\hline Total & $15(3.2)$ & $25(5.4)$ & $192(41.2)$ & $232(49.8)$ & 0.00 \\
\hline
\end{tabular}

Categories are not mutually exclusive. 
ities on conventional radiographs. The radiographs and the findings on the CRF were not available at that time.

We divided the completed CRFs over two groups: group A had no history of trauma, and group B had a history of trauma of more than 4 weeks before presentation at the hospital.

We used the Chi-squared test to identify significant differences between the yield of radiographic and MR diagnoses, and between the number of findings in group A and that in group B. To look for statistically significant differences between the yield of radiographs and MRI within groups A, B and the study population as a whole, we used McNemar's Chi-squared test.

\section{Results}

The median age of the 798 patients included was 31 years (range 16-45 years), and 261 patients (32.7\%) were female.
Fig. 1 Severe osteoarthritis on both radiographs and MRI. a, b Lateral and antero-posterior radiographs showing deformation of the femoral condyles and tibia plateau with marked subchondral cyst formation and osteophyte formation in a 44-year-old female patient with a history of knee trauma (group B). c-e Coronal proton density and T2-weighted spin-echo (SE) images and of the same lesion showing, in addition to the deformities and changes already noted on the radiographs, the osteochondral defect to a better extent. e Sagittal T2-weighted SE image showing the same features, but also focal fullthickness cartilage loss
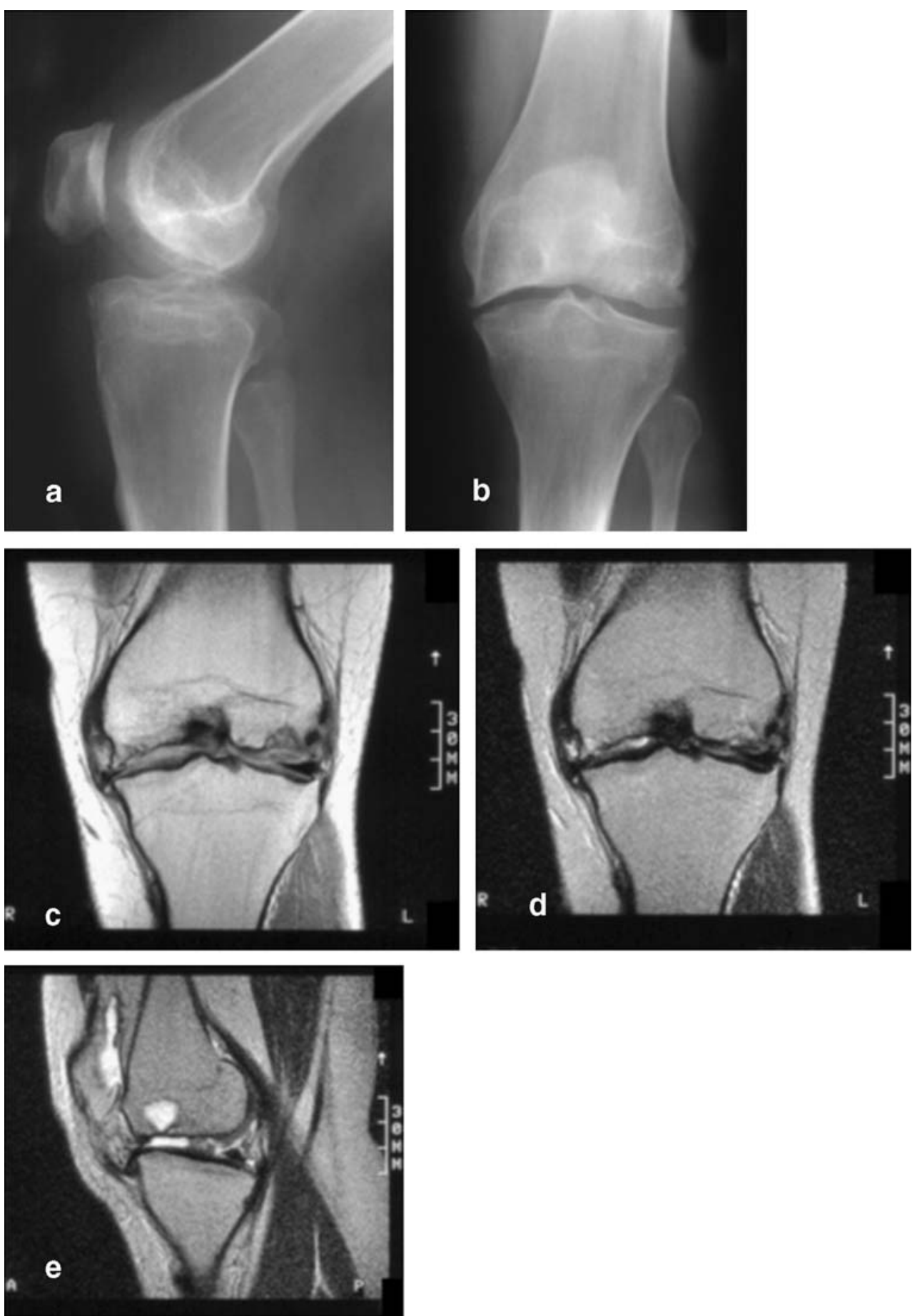
Minimal and median duration of symptoms were 4 and 20 weeks respectively (range 4-1,490 weeks). Three hundred and thirty-two patients reported no history of trauma (group A), and 466 patients reported an old (more than 4 weeks prior to consultation) history of trauma (group B).

Magnetic resonance imaging depicted arthroscopically treatable intra-articular pathology in 341 patients $(42.7 \%)$. Medial and lateral meniscal tears were found in 225 (28.2\%) and 111 patients (13.9\%) respectively. The average radiation dose of supine antero-posterior and lateral radiographs was 0.2 and $0.3 \mathrm{mSv}$ respectively. The median duration of the interval between radiographs and MRI was 8 days (95 percentile, 85 days). In 196 patients (24.6\%) radiographs were obtained prior to inclusion in the study. These radiographs were not repeated since the knee complaints had not changed between the moment they were obtained and inclusion in this study.

In group $\mathrm{A}$, radiographs showed 36 osseous abnormalities in 332 patients $(10.8 \%$, Table 2). All these abnormal- ities, except 13 Kellgren [1] grade 1 osteoarthritis cases, were also depicted on MRI. There were 23 concordant radiographic and MRI diagnoses (Fig. 3; Table 2). One incidental finding (enchondroma) was demonstrated on radiographs and MRI. The majority of the 72 diagnoses made only with MRI were osteoarthritis and bone bruising (Table 2). Most osseous lesions (osteoarthritis, articular surface lesions, loose bodies and bone marrow edema) with the exception of incidental findings (one case) and old fractures (not present in this group) were significantly more often diagnosed with MRI than with radiographs (Table 2).

In group B, radiographs showed 40 osseous abnormalities in 466 patients $(8.6 \%$, Table 3$)$. All these abnormalities, except 15 Kellgren [1] grade 1 osteoarthritis cases, were also depicted on MRI. There were 25 concordant radiographic and MRI diagnoses (Figs. 1, 4, 5 and 6; Table 3). Four of the six incidental findings were diagnosed on radiographs and MRI (1 non-ossifying fibroma and 3 enchondromas, Fig. 7). Two enchondromas were depicted
Fig. 2 Three-month-old transchondral fracture on both radiographs and MRI. a, b Antero-posterior and lateral radiograph showing irregularity and deformation of the lateral femoral condyle consistent with a transchondral fracture of the lateral femoral condyle in a 40-year-old male with a history of knee trauma (group B). c-d Sagittal T2-weighted SE images and $\mathrm{T} 1$-weighted $3 \mathrm{D}$ gradientecho (GE) with fat suppression showing subtle contour deformity of the lateral femoral condyle with kissing bone bruises in the lateral femoral condyle and tibial plateau
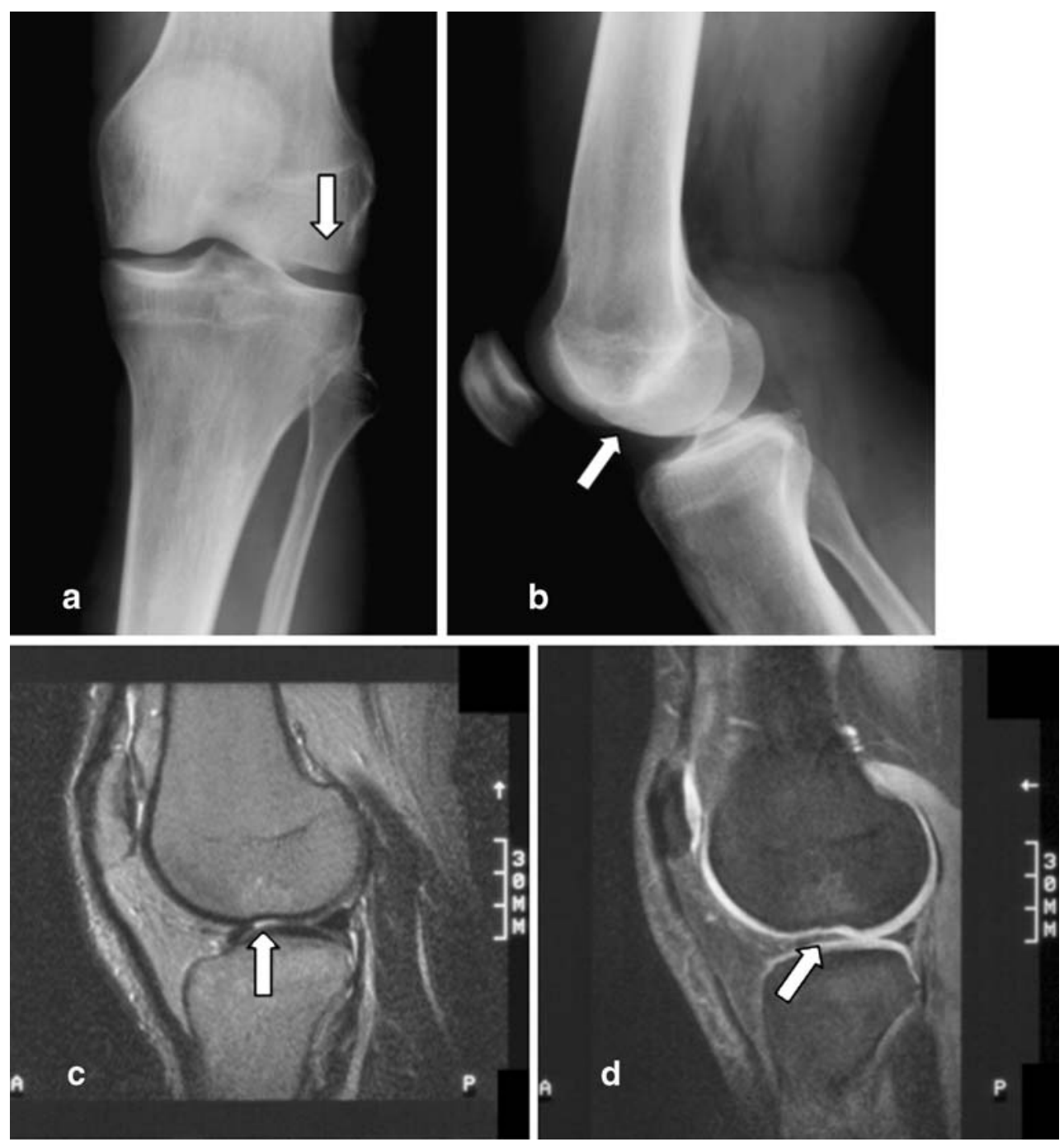
Table 4 Osseous abnormalities detected on radiographs only, on radiographs and MRI, and on MRI only in 798 patients with non-acute knee complaints

\begin{tabular}{|c|c|c|c|c|c|}
\hline Osseous abnormality & $\mathrm{R}(\%)$ & $\mathrm{R}$ and MRI (\%) & MRI (\%) & Total $(\%)$ & McNemar test, $p$ value \\
\hline $\mathrm{OA}$ & $28(3.5)$ & $31(3.9)$ & $104(13.0)$ & $163(20.4)$ & 0.00 \\
\hline ASL & $0(0)$ & $6(0.8)$ & $29(3.6)$ & $35(4.4)$ & 0.00 \\
\hline LB & $0(0)$ & $20.3)$ & $10(1.3)$ & $12(1.5)$ & 0.00 \\
\hline Fractures & $0(0)$ & $4(0.5$ & $3(0.4)$ & $7(0.9)$ & 0.25 \\
\hline Incidental finding & $0(0)$ & $5(0.6)$ & $2(0.3)$ & $7(0.9)$ & 0.5 \\
\hline Bone marrow edema & $0(0)$ & $0(0)$ & $96(12.0)$ & $96(12.0)$ & NA \\
\hline Total & $28(3.5)$ & $48(6.0)$ & $264(33.1)$ & $340(42.6)$ & 0.00 \\
\hline
\end{tabular}

Categories are not mutually exclusive.

on MRI only (Fig. 8). The majority of the 192 diagnoses made only with MRI were bone marrow edema (Fig. 2), osteoarthritis and articular surface lesions (Table 3). These three diagnoses were significantly more often diagnosed with MRI than with radiographs (Table 3). There was no significant difference between MRI and radiographs in the diagnosis of loose bodies, old fractures and incidental findings.

Fig. 3 Twenty-six-month-old transchondral fracture on both radiographs and MRI. a Anteroposterior radiograph showing abnormal density, subtle irregularity and deformation of the medial femoral condyle in a 35-year-old male patient with no history of knee trauma (group A). b-d Sagittal T1-weighted 3D GE with fat suppression and coronal proton density and T2-weighted SE images of this knee showing the transchondral fracture
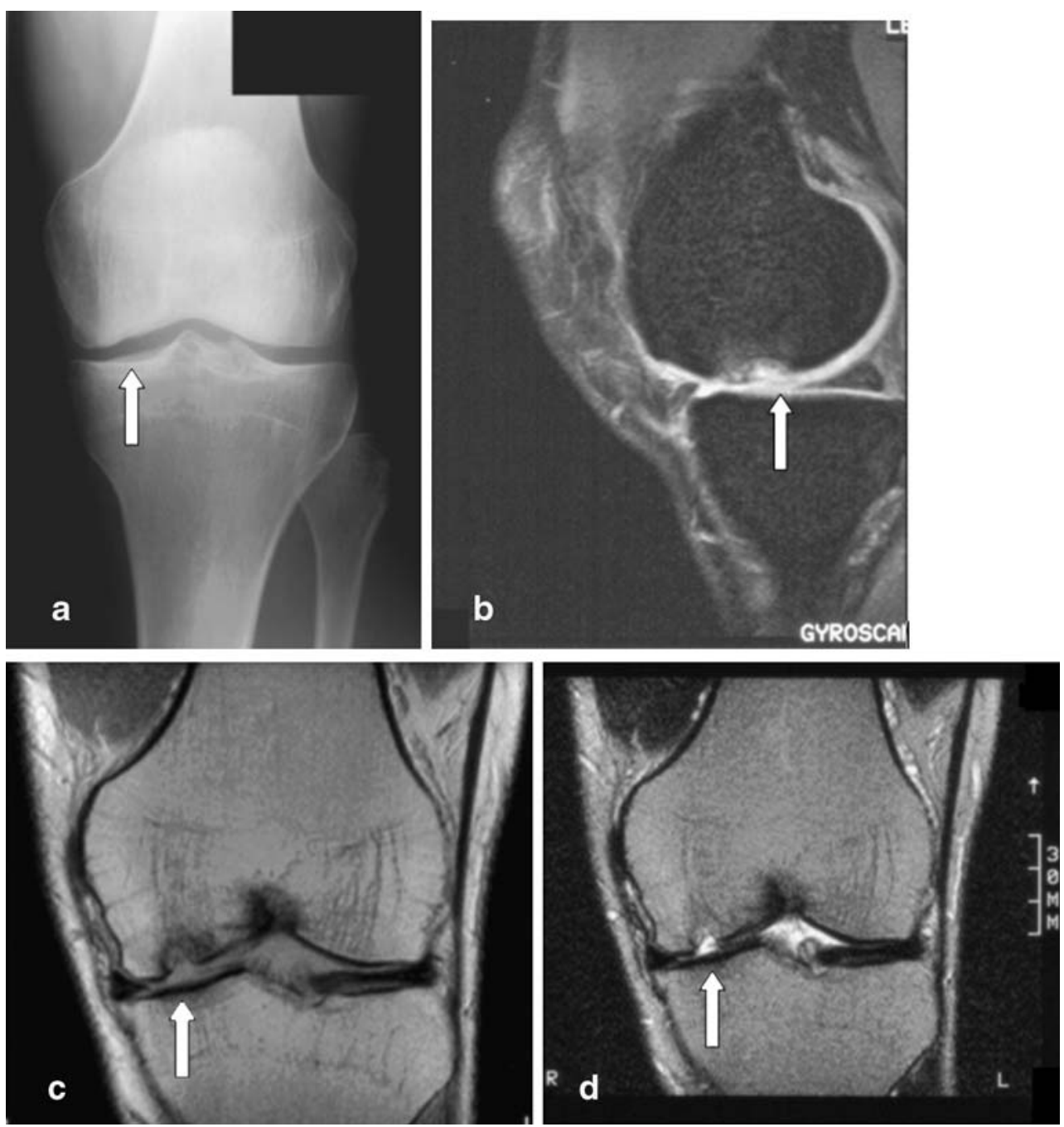

Osseous abnormalities were more frequently encountered in group B than in group A $(p<0.001)$. This difference is explained by a significant difference in the number of old fractures ( $p<0.05$, no old fractures were seen in group A), and patients with bone marrow edema $(p<0.001)$.

Bone marrow edema was demonstrated with MRI in 116 patients (14.5\%, Table 4); 96 of these were found in group B $(82.8 \%$ of patients with bone marrow edema, Table 3$)$. 
In $6(30 \%)$ of the 20 group A patients with bone marrow edema, it was either associated with intra-articular damage of non-osseous origin or it was an isolated finding. The associated osseous abnormalities in the other 14 patients were as follows: bone marrow edema was found in 4 of the 10 patients with articular surface lesions, in 1 of the 7 patients with a loose body, and in 9 of the 70 patients with osteoarthritis.

In $89(92.7 \%)$ of the 96 group B patients with bone marrow edema, it was either associated with intra-articular damage of non-osseous origin or it was an isolated finding. The associated osseous abnormalities in the other 7 patients were as follows: bone marrow edema was found in 4 of the 25 patients with articular surface lesions (Fig. 2), in 1 of the
5 patients with a loose body and in 2 of the 93 patients with osteoarthritis.

In our population we did not find any other osseous lesions like osteopenia or femoropatellar disease. Also, we did not find chondrocalcinosis on radiographs.

Subsequently, all patients with radiographically detected abnormalities with the exception of Kellgren [1] grade 1 and 2 osteoarthritis were identified. MRI studies of the 17 patients with 18 radiographically detected osseous abnormalities were mixed with 17 gender- and age-matched MRI studies of patients without abnormalities on radiographs. There were 6 females in each group, and the mean age in each group was 33.1 years, SD 7.9 years). At revision, the

Fig. 4 Loose body on both radiography and MRI. a Lateral radiograph showing a ventrally located loose body in the left femorotibial joint in an 18-yearold male professional skater with a history of knee trauma (group B). b-c Sagittal T1-weighted 3D GE with fat suppression and coronal proton density SE images of the same patient, also showing the loose body that is ventrally located in the lateral compartment of the femorotibial joint. At subsequent arthroscopy this loose body was removed
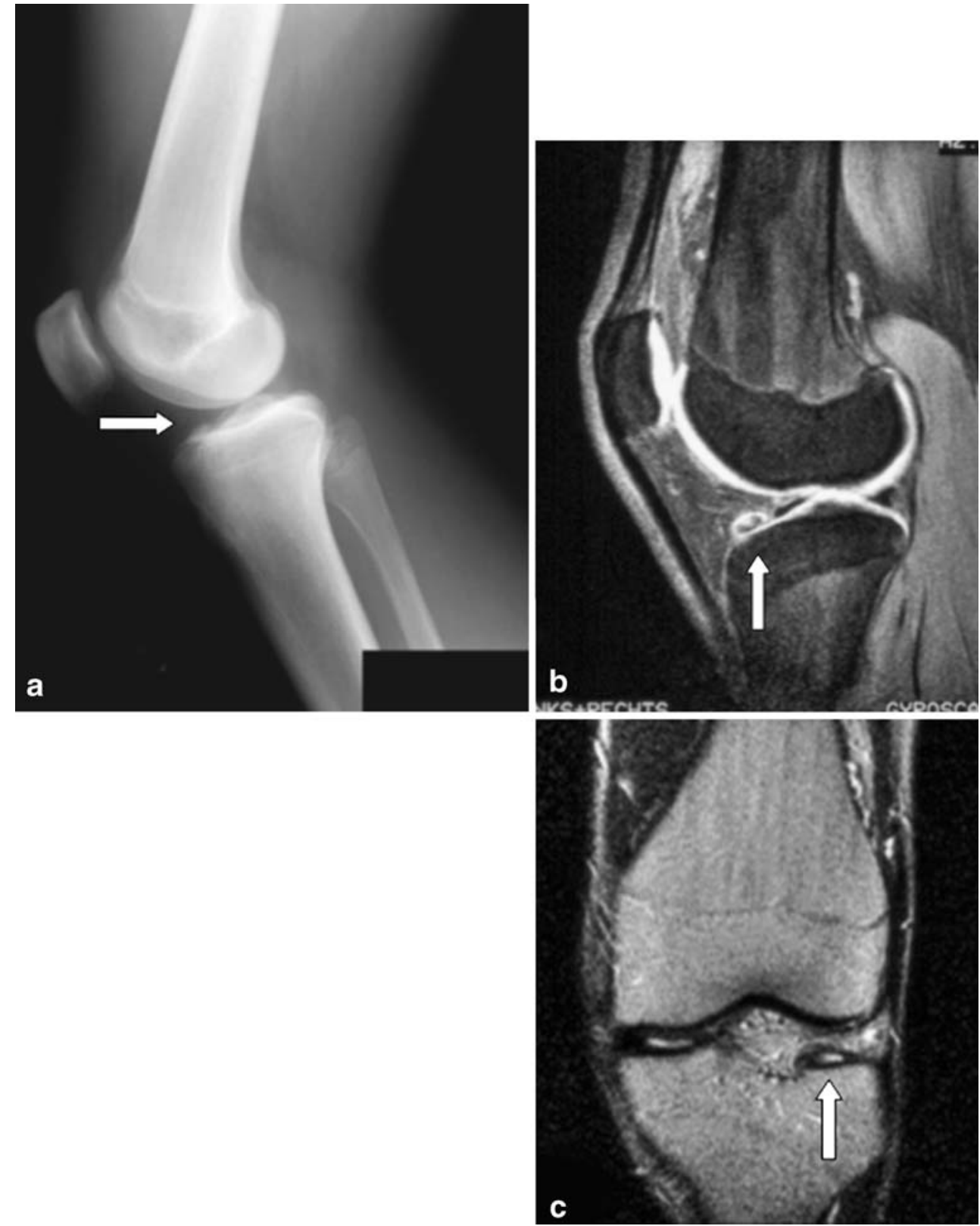
Fig. 5 Eight-month-old healed fracture on both radiograph and MRI. a Lateral radiograph showing a healed fracture of the inferior patellar pole in an 18 -year-old male patient with a history of knee trauma (group B). b Sagittal proton density SE image of the same patient, also delineating the healed fracture of the inferior pole of the patella

Fig. 6 Two-month-old healing fracture on both radiograph and MRI. a Lateral radiograph showing a healing fracture of the left inferior patellar pole in a 40 -year-old male patient with a history of knee trauma (group B). b, c Sagittal T1-weighted 3D GE with fat suppression and T2weighted SE images of the same patient, also delineating the healing fracture of the inferior pole of the patella without cartilaginous irregularities. Around the fracture line bone marrow edema can be appreciated
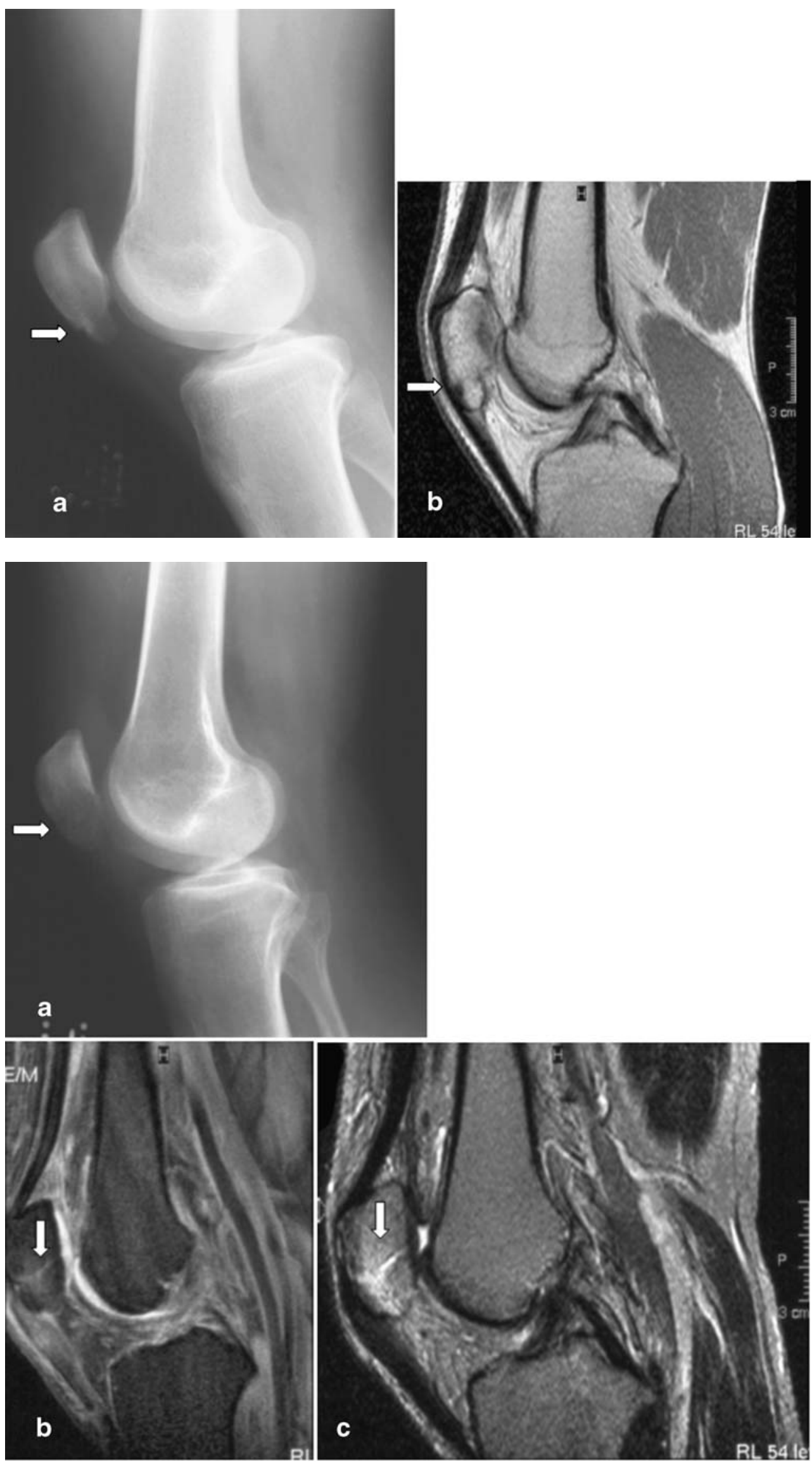
reader (JLB) was blinded to all information and had no access to the radiographs. All 18 osseous abnormalities (1 caae of osteoarthritis Kellgren grade 3 [Fig. 1], 6 articular surface lesions [Figs. 2, 3], 2 loose bodies [Fig. 4], 4 old healed fractures [Figs. 5, 6] and 5 incidental findings [4 enchondromas and 1 non-ossifying fibroma, Figs. 7, 8]) were all diagnosed on MRI.

\section{Discussion}

Key characteristics of our population such as age, gender, clinical suspicion of intra-articular abnormalities, intraarticular abnormalities detected with MRI, and history suggest that our conclusions can be applied to typical populations scheduled for MRI to analyze non-acute knee problems.

The yield of radiographs in patients with subacute or chronic knee symptoms who are otherwise healthy and who are scheduled for MRI based on the results of a physical exam suggesting the presence of intra-articular damage is only $9.5 \%$ (76 patients). The majority of these patients (77.6\%) have osteoarthritis. We failed to confirm with MRI the radiographic diagnosis of Kellgren [1] grade 1 osteoarthritis in 28 patients, but none of the other abnormalities were diagnosed only with radiographs. This was confirmed in a second reading of MRI blinded to clinical and radiographic information. Radiographs do not, in the setting of this study, contribute to clinical decisionmaking or clinical management of the patient. Although the dose administered when obtaining radiographs of the knee is small, the size of the population with knee complaints make the reduction of an administered dose a relevant issue. Radiographs can therefore be omitted in this specific population when the decision to perform MRI is taken based on clinical assessment. The disadvantage of occasionally missing Kellgren [1] grade 1 osteoarthritis is limited when we realize that because of the lack of a gold
Fig. 7 Incidental finding depicted on both radiographs and MRI. a, b Lateral and antero-posterior radiographs showing a central, metaphyseally located lesion with chondroid matrix consistent with enchondroma in the femur in a 44-year-old female patient with a history of knee trauma (group B). c, d Sagittal proton density and T2-weighted SE images of the same lesion
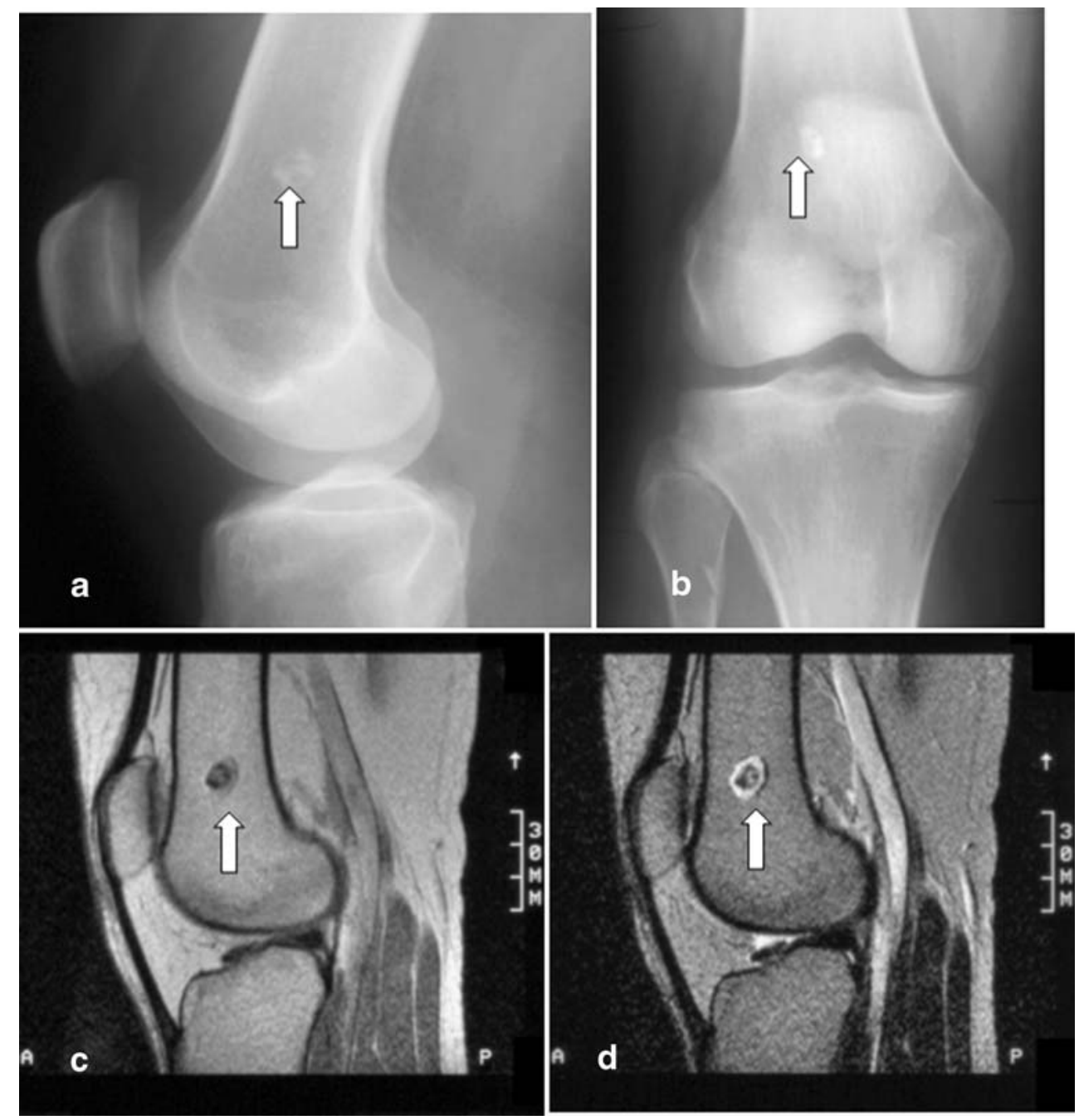
Fig. 8 Incidental finding depicted on MRI only. a Lateral radiograph showing no femoral osseous lesion in a 40-year-old female patient with a history of knee trauma (group B). b, c Sagittal proton density and T2-weighted SE images showing a metaphyseal lesion consistent with an enchondroma
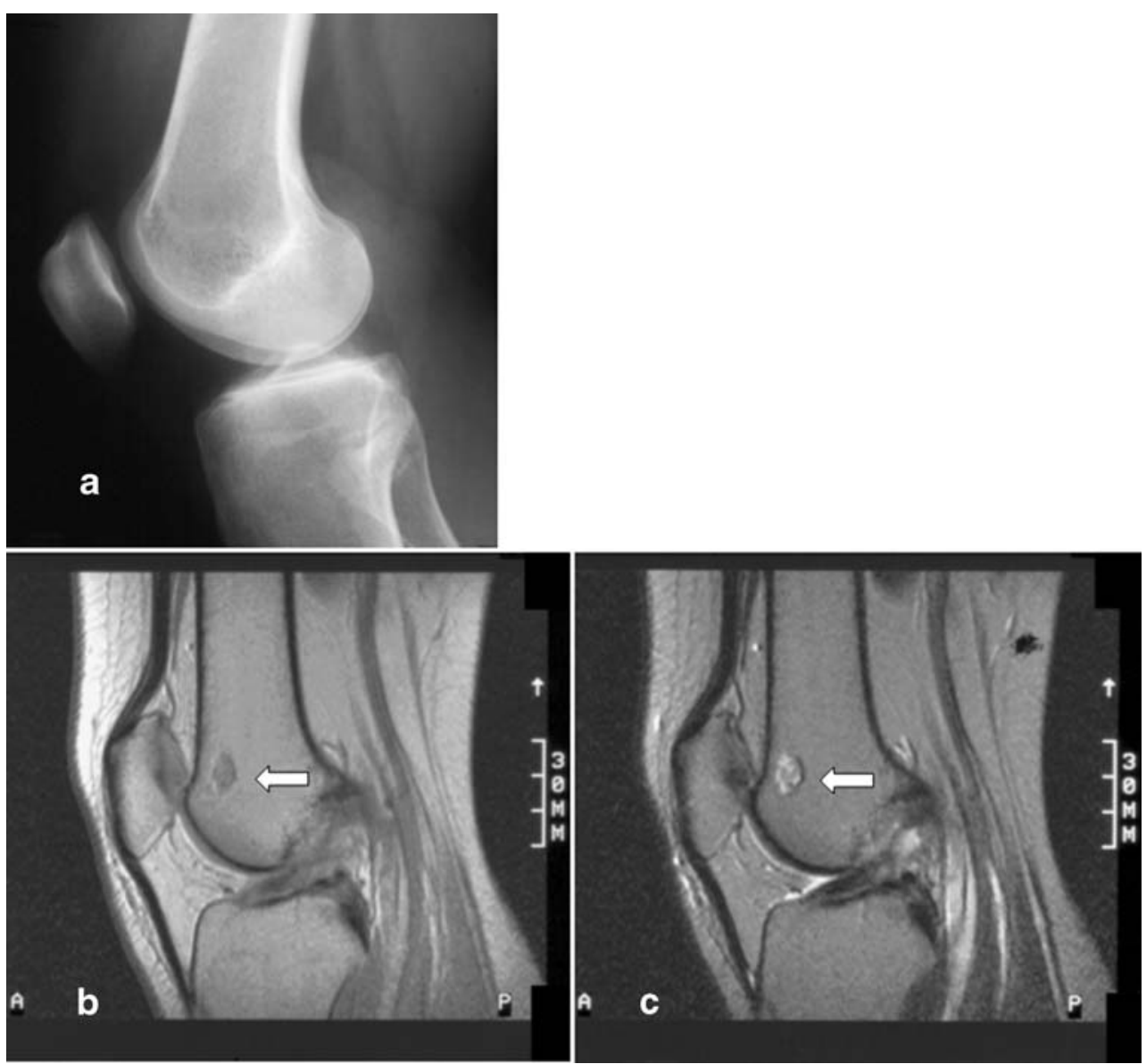

standard these radiographic diagnoses may also be falsepositive.

Taljanovic et al. [6] rated radiographs as "essential" or "very important" in $46-58 \%$ of cases and radiographs added "not needed information" in $14-29 \%$ of cases. They conclude in their study that radiographs are an important, and sometimes essential initial, complementary study for reading musculoskeletal MR examinations. This apparently different conclusion can be explained by the differences in study populations. Taljanovic et al. [6] analyzed a large variety of diagnosis (trauma, infection, tumors, degenerative or miscellaneous) in all anatomical locations in patients of all ages. We only analyzed patients between 16 and 45 years of age with non-acute knee symptoms lasting at least 4 weeks, excluding amongst others patients with acute knee symptoms (i.e., less than 4 weeks), history of rheumatoid arthritis, and clinical diagnosis of retropatellar chondromalacia. Other major methodological differences are mainly related to this difference.

The concordance between radiographs and MRI is not high because of the large number of diagnoses (77.6\%) made only with MRI. In groups A and B osteoarthritis, articular surface lesions, and bone marrow edema were significantly more often diagnosed with MRI than with radiographs. Loose bodies were significantly more frequently diagnosed with MRI in group A only. This higher yield of MRI is no surprise, since abnormalities such as articular surface lesions and bone marrow edema are known to be better, or even exclusively appreciated on MRI. These findings have, in addition to the intra-articular abnormalities detected with MRI, potential clinical significance in view of the patients' presenting symptoms.

The advantage and comprehensiveness of MRI is illustrated by its ability to demonstrate bone marrow edema that is associated with other abnormalities. In the patients with a history of old trauma, bone marrow edema was mainly $(92.7 \%)$ associated with intra-articular damage. In the group with no history of trauma, however, bone marrow edema was mainly (70\%) seen in association with osseous abnormalities, thus facilitating the diagnosis of articular surface lesions and osteoarthritis.

This prospective study has several limitations that are mainly related to the decision to follow usual care. This means that radiographs were available at the time of MRI. To overcome this limitation, one radiologist (JLB) reevaluated MR studies of patients with abnormalities on conventional radiographs without knowledge of the radiographs, as described. 
Another limitation is that we could not determine accuracy because of the absence of a reference standard. If we look at the discordant cases, there are no potentially false-negative MRI diagnoses (with the exception of Kellgren [1] grade 1 osteoarthritis), but the number of potential false-positive MRI diagnoses (abnormal finding on MRI in combination with a normal radiograph) is substantial, mainly because of the high prevalence of bone marrow edema.

Another possible limitation of this study is the selection bias introduced by including only patients between 16 and 45 years of age and excluding patients with co-morbidity. The study design was not tailored toward assessment of the value of conventional radiographs only. The overall goal of this multicenter study was to evaluate the efficacy of MRI compared with direct arthroscopy in patients with subacute knee complaints, based on clinical outcome. To this end we tried to obtain a homogenous study population without comorbidity. This is most likely the reason we encountered only 1 patient with high-grade osteoarthritis (Kellgren [1] grade 3). However, because the majority of MR examinations performed in our institutions will be of patients in this age range without known co-morbidity, we do not think this selection bias will alter our overall conclusions.

We tried to mimic usual care as much as possible in this study. However, in our study MRI was performed within 2 weeks of inclusion in the study. Due to the limited availability of MRI compared with conventional radiographs in most hospitals, MRI often cannot be performed at such short notice. This can lead to a considerable waiting time for MR examinations and this may direct physicians to prior conventional radiographs to rule out gross pathology and reassure the patient. To prevent these unnecessary radiographs, the waiting time for MRI must be as short as possible. In the Netherlands waiting times for MRI in most institutions are down from several months a couple of years ago to several weeks nowadays.

We conclude that radiographs should not be obtained routinely when MRI is being performed in non-acute young patients with knee complaints because the number of clinically relevant lesions detected on radiographs is low. In addition, the small number of abnormalities found on radiographs is, with the exception of an occasional case of
Kellgren [1] grade 1 osteoarthritis, also depicted on MRI. Reduction of costs and radiation dose is small in the individual patient, but, because of its size, substantial in the entire population with subacute and chronic complaints suspected to be knee injury.

\section{References}

1. Kellgren JH, Jeffrey M, Ball J. Atlas of standard radiographs. Oxford: Blackwell Scientific, 1963.

2. Bauer SJ, Hollander JE, Fuchs SH, Thode HC Jr. A clinical decision rule in the evaluation of acute knee injuries. J Emerg Med 1995; 13(5): 611-615.

3. Seaberg DC, Jackson R. Clinical decision rule for knee radiographs. Am J Emerg Med 1994; 12(5): 541-543.

4. Stiell IG, Wells GA, Hoag RH et al. Implementation of the Ottawa Knee Rule for the use of radiography in acute knee injuries. JAMA 1997; 278(23): 2075-2079.

5. American College of Radiology ACR. Appropriateness Criteria 2000. Radiology 2000; 215(Suppl): 1-1511.

6. Taljanovic MS, Hunter TB, Fitzpatrick KA, Krupinski EA, Pope TL. Musculoskeletal magnetic resonance imaging: importance of radiography. Skeletal Radiol 2003; 32(7): 403-411.

7. Bohndorf K. Imaging of acute injuries of the articular surfaces (chondral, osteochondral and subchondral fractures). Skeletal Radiol 1999; 28(10): 545-560.

8. Lee JK, Yao L. Stress fractures: MR imaging. Radiology 1988; 169(1): 217-220.

9. Meyers SP, Wiener SN. Magnetic resonance imaging features of fractures using the short tau inversion recovery (STIR) sequence: correlation with radiographic findings. Skeletal Radiol 1991; 20 (7): 499-507.

10. Mink JH, Deutsch AL. Occult cartilage and bone injuries of the knee: detection, classification, and assessment with MR imaging. Radiology 1989; 170(3 Pt 1): 823-829.

11. Manaster BJ. Tumors/other connective tissue tumors: fibrous origin/nonossifying fibroma/benign fibrous cortical defect. In: Manaster BJ, Disler DG, May DA, editors. Musculoskeletal imaging. St Louis: Mosby; 2002. 70-71.

12. Manaster BJ. Tumors/cartilage forming tumors: benign/enchondroma. In: Manaster BJ, Disler DG, May DA, editors. Musculoskeletal imaging. St Louis: Mosby; 2002. 37-42.

13. Ryu KN, Jin W, Ko YT et al. Bone bruises: MR characteristics and histological correlation in the young pig. Clin Imaging 2000; 24(6): 371-380.

14. Recht MP, Kramer J, Marcelis S et al. Abnormalities of articular cartilage in the knee: analysis of available MR techniques. Radiology 1993; 187(2): 473-478. 